\title{
Harmonic Injection PWM of Multiple Inverter for Ripple Torque Reduction
}

\author{
Member Satoru Ito \\ (Hitachi, Ltd.) \\ Member Kiyoshi Nakata \\ (Hitachi, Ltd.) \\ Member Shigeta Ueda \\ (Hitachi, Ltd.) \\ Member Kiyoshi Nakamura (Hitachi, Ltd.)
}

In this paper, a novel harmonic injection PWM method is proposed for harmonic reduction. The third harmonic is injected to the reference voltage, of which amplitude and phase angle are regulated so that the dominant ripple torque components are reduced.

In a large capacity power converter, GTOs are commonly used as the switching devices and a multiple configuration of unit power converters has been applied. The use of GTOs allows higher voltage and larger current to be handled. However, it results in increased harmonics, because of their relatively low switching frequency of about $500 \mathrm{~Hz}$. Therefore, harmonic reduction method for large capacity applications is of great interest, which is suitable for a multiple power converter without increasing the cost. The harmonic injection PWM fulfills the requirement.

The harmonic components of output voltage, output current and torque when a harmonic component is injected are analyzed in detail. The most suitable amplitude and phase angle are derived from theoretical analysis. Simulation results show the effectiveness of the method.

Key words : PWM, Multiple power converter, Harmonic, Ripple torque, Harmonic injection

\section{Introduction}

These days, power converters are widely used for their high efficiency and controllability ${ }^{(1)}$. Even large capacity applications such as rolling mill drives $^{(2)(3)}$, converters for $\mathrm{SVCs}^{(4)}$, and inverters of magnetically levitated transportation system ${ }^{(5)-(8)}$, can make use of power converters of multiple configuration.

In most large capacity application, GTOs are used as switching devices, often in series connection. For their relatively low switching frequency, a large amount of harmonics is generated. Harmonic voltage components cause current harmonics, and ripple torque in the case of a motor drive. They result in noise, vibration and heat loss of the load. Therefore, reduction of the harmonics is of great interest $^{(5) \sim(7)}$.

There are several approaches to reduce harmonics. Multiple inverters of configurations where larger numbers of unit inverters are connected in one phase contribute to the harmonic reduction. On the other hand, such configurations increase cost and size of the inverters, sometimes impractically.

PWM methods using space vectors are effective to reduce harmonics and suitable for digital control circuit $^{(9)(10)}$. But it is difficult to apply them to a multiple inverter, because the number of output voltage vectors is so large that a much longer time is needed to choose the suitable output vector.

Some PWM methods with harmonic injection have also been proposed ${ }^{(11)-(13)}$. In Refs (11) and (12), the third harmonic is added to the sinusoidal reference in order to increase the maximum output 
voltage of the inverter, which results in harmonic reduction. In Ref (13), a rectangular waveform is injected and the amplitude is controlled so that the harmonics are reduced.

In Refs (5) and ( 6 ), harmonic characteristics of multiple inverters using subharmonic method are investigated using theoretical analysis and digital simulation. In this paper, a novel injection PWM is proposed, where the third harmonic is injected into the sinusoidal reference voltage, for which the amplitude and phase angle are actively controlled so that the harmonic components are reduced. First, the relations between harmonic components of voltage, current and torque are investigated when a harmonic component is injected into the sinusoidal reference. Next, suitable conditions of the injected component needed to reduce ripple torque are clarified. Then, simulation results are given showing the effectiveness of the method.

\section{Main circuit configuration}

Fig. 1 shows the main circuit configuration of a multiple inverter. A synchronous motor (SM) is connected to the multiple inverter, which consists of twelve full-bridge inverters (three phases, four

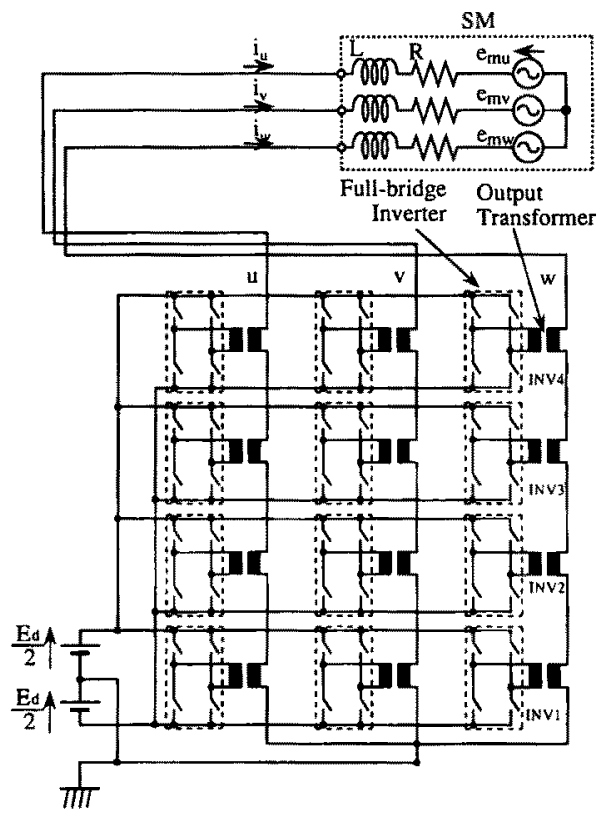

Fig. 1. Main circuit configuration. stages), sharing a common DC source. The secondary windings of the transformers of each phase are connected in series, and the sum of the output voltage of each full-bridge inverter is the output voltage of the multiple inverter.

\section{Analysis of waveforms generated using the subharmonic method}

Fig. 2 shows the minimum unit of a voltage source inverter, which is called a unit inverter in this paper. Fig. 3 shows the basic strategy of the subharmonic method for a unit inverter. The switches turn on and off every time the modulation wave $y_{m}$ and the carrier wave $y_{c}$ intersect. As a result, the output voltage becomes a pulse train, of which pulse width varies depending on the modulation wave.

The output voltage of the unit inverter, $e$, can be decomposed using a Fourier series ${ }^{(5)(14)}$ :

$$
\begin{aligned}
e= & \frac{E_{d} A_{1}}{2} \sin \left(\omega_{1} t+\theta_{1}\right) \\
& +E_{d} \sum_{m} \sum_{k} S_{(m, k)} \sin \left(\omega_{(m, k)} t+\theta_{(m, k)}\right) \\
& +E_{d} \sum_{m} \sum_{k} C_{(m, k)} \cos \left(\omega_{(m, k)} t+\theta_{(m, k)}\right)
\end{aligned}
$$

where,

$S_{(m, k)}, C_{(m, k)}$ : amplitude indices of harmonic components shown in Table 1

$$
\omega_{1}=2 \pi f_{1}
$$

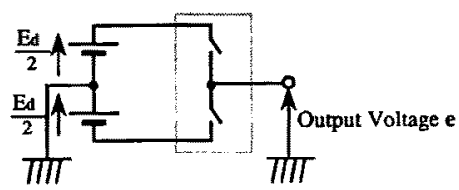

Fig. 2. Configuration of unit inverter.

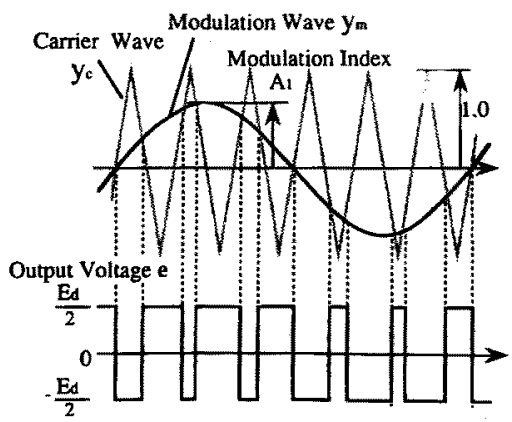

Fig. 3. Principle of subharmonic method. 
$\omega_{(m, k)}=2 \pi f_{(m, k)}$

$f_{(m \cdot k)}=m f_{c}+k f_{1}$

$\theta_{(m, k)}=m \theta_{c}+k \theta_{1}$

$m, k:$ integers

$E_{d}: \mathrm{DC}$ voltage

$A_{1}$ : modulation index

$f_{1}$ : inverter output frequency

$\theta_{1}:$ phase angle of modulation wave

$f_{c}$ : carrier frequency

$\theta_{c}$ : phase angle of carrier wave

The output voltage contains higher order harmonics, of which amplitudes, frequencies and phase angles are described using the integers $m$ and $\boldsymbol{k}$, as is shown in Table 1.

The output voltage of the multiple inverter in Fig. 1 can be considered the sum of those generated by eight unit inverters as in Eq. (1). For harmonic redution, all the unit inverters are given the same modulation wave, and the phase angles of triangular carrier waves are shifted at equal intervals $\Delta \theta^{(5)}$ :

$$
\Delta \theta=360 / 2 N \quad(\mathrm{deg})
$$

where multiple index $N$ is defined as the number of full-bridge inverters in one phase.

Therefore, the output voltage of the multiple inverter, $e_{s}$, can be expressed as :

$$
\begin{aligned}
e_{s}= & w N E_{d} A_{1} \sin \left(\omega_{i} t+\theta_{1}\right) \\
& +w N E_{d} \sum_{m} \sum_{k} A_{(m, k)} \sin \left(\omega_{(m, k)} t+\theta_{(m, k)}\right)
\end{aligned}
$$

where

$w$ : winding ratio of output transformers

$N$ : number of full-bridge inverters in one phase

\begin{tabular}{|c|c|c|}
\hline$m$ & $k$ & $S_{(m, k)}$ \\
\hline $2,4,6, \cdots$ & $\begin{array}{l} \pm 1, \pm 3 \\
\pm 5, \cdots\end{array}$ & 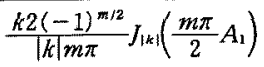 \\
\hline
\end{tabular}

Table 1. Amplitude indices of harmonic components in Eq. (1).

\begin{tabular}{c|c|c} 
(b) $C_{(m, k)}$ & \\
\hline$m$ & $k$ & $C_{(m, k)}$ \\
\hline $1,3,5, \cdots$ & $\begin{array}{c}0, \pm 2, \\
\pm 4, \cdots\end{array}$ & $\frac{2(-1)^{(m+1) / 2}}{m \pi} J_{|k|}\left(\frac{m \pi}{2} A_{t}\right)$ \\
\hline \multicolumn{2}{c}{ other combinations } & 0 \\
\hline
\end{tabular}

$J_{n}(x)$ : Bessel function of first kind of order $n$

電学論D, 113 巻 9 号, 平成 5 年
$A_{(m, k)}$ : amplitude index of harmonic component shown in Table 2

$m, k:$ integers

In Eq. ( 3 ), harmonic components are located in the neighborhood of the equivalent carrier frequency $f_{C E}$. The frequency of a harmonic component is expressed as :

Table 2. Amplitude index of harmonic components in Eq. ( 3 ).

\begin{tabular}{c|c|c}
\hline$m$ & $k$ & $A_{(m, k)}$ \\
\hline $2 N, 4 N$, & $\pm 1, \pm 3$, & $\frac{k 4(-1)^{m / 2}}{|k| m \pi} J_{|\kappa|}\left(\frac{m \pi}{2} A_{1}\right)$ \\
$6 N, \cdots$ & $\pm 5, \cdots$ & 0 \\
\hline \multicolumn{2}{l}{ other combinations } \\
\hline
\end{tabular}

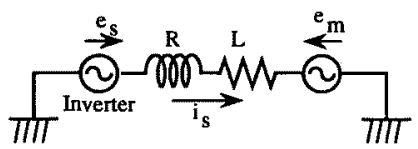

(a) Equivalent for main circuit.

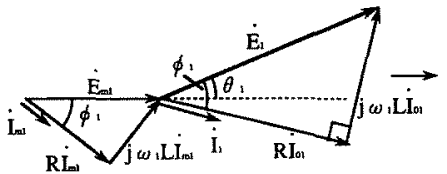

$\dot{I}_{a 1}$ : current component generated by $E_{1}$ $\dot{I}_{m 1}$ : current component generated by $E_{m 1}$

(b) Vector diagram for fundamental component.



$\left|\dot{E}_{(m, k)}\right|=\frac{w N E_{\alpha} A_{(m, k)}}{\sqrt{2}}$

$\dot{I}_{(m, n)}$ : current component generated by $\dot{E}_{(m, k)}$

(c) Vector diagram for harmonic components.

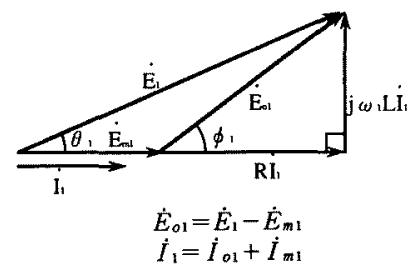

(d) Vector diagram for fundamental component when it is controlled in phase with induced voltage.

Fig. 4. Main circuit model and vector diagrams. 
$f=g f_{C E}+l f_{1}$

where $f_{C E}=2 N f_{C}, g=1,2,3,4,5, \cdots, l= \pm 1, \pm 3$, $\pm 5, \cdots$

In order to derive the harmonic current, the main circuit model of Fig. 4 (a) is supposed. For simplicity, the neutral point of the load is grounded and the induced voltage of SM has only the fundamental component. The fundamental and the harmonic components of the output current can be derived using vector diagrams Figs 4 (b) and (c) :

$$
\begin{aligned}
i_{s}= & \frac{1}{Z_{1}} w N E_{d} A_{1} \sin \left(\omega_{1} t+\theta_{1}-\phi_{1}\right) \\
& -\frac{1}{Z_{1}} \sqrt{2} E_{m} \sin \left(\omega_{1} t-\phi_{1}\right) \\
& +\frac{w N E_{d}}{Z_{(m, k)}} \sum_{m} \sum_{k} A_{(m, k)} \\
& \times \sin \left(\omega_{(m, k)} t\right. \\
& \left.+\theta_{(m, k)}-\phi_{(m, k)}\right)
\end{aligned}
$$

where

$$
\begin{aligned}
& Z_{1}=\sqrt{R^{2}+\left(\omega_{1} L\right)^{2}} \\
& \phi_{1}=\tan ^{-1}\left(\omega_{1} L / R\right) \\
& Z_{(m, k)}=\sqrt{R^{2}+\left(\omega_{(m, k)} L\right)^{2}} \\
& \phi_{(m, k)}=\tan ^{-1}\left(\omega_{(m, k)} L / R\right) \\
& e_{m}=\sqrt{2} E_{m} \sin \left(\omega_{1} t\right)
\end{aligned}
$$

Also for simplicity, the fundamental component of the output current is supposed to be controlled in phase with the induced voltage. Then, the fundamental output voltage, modulation index and phase angle of the modulation wave, and output current components can be expressed using the vector diagram in Fig. 4 (d) :

$$
\left.\begin{array}{rl}
E_{1}= & \sqrt{\left(E_{m 1}+R I_{1}\right)^{2}+\left(\omega_{1} L I_{1}\right)^{2}} \\
E_{o 1} & =\sqrt{\left(R I_{1}\right)^{2}+\left(\omega_{1} L I_{1}\right)^{2}} \\
A_{1} & =\frac{\sqrt{2} E_{1}}{w N E_{d}} \\
\theta_{1}= & \tan ^{-1}\left\{\omega_{1} L I_{1} /\left(E_{m 1}+R I_{1}\right)\right\} \\
i_{s}= & \frac{1}{Z_{1}} \sqrt{2} E_{o 1} \sin \left(\omega_{1} t\right) \\
& +\frac{w N E_{d}}{Z_{(m, k)}} \sum_{m} \sum_{h} A_{(m, k)} \\
& \times \sin \left(\omega_{(m, k)} t\right. \\
& \left.+\theta_{(m, k)}-\phi_{(m, k)}\right)
\end{array}\right\}
$$

where

$I_{1}$ : fundamental component of output current
From Eq. (6), the frequencies of the harmonic current components are the same as those of the harmonic voltage in Eq. (3).

The output currents of the three phases $i_{u}, i_{v}$ and $i_{w}$ are derived by making the phase angle $\theta_{(m, k)}$ in Eq. (6) as follows:

$$
\begin{aligned}
& i_{u}: \theta_{(m, k)}=m \theta_{c}+k \theta_{1} \\
& i_{v}: \theta_{(m, k)}=m \theta_{c}+k\left\{\theta_{1}-(2 / 3) \pi\right\} \\
& i_{w}: \theta_{(m, k)}=m \theta_{c}+k\left\{\theta_{1}-(4 / 3) \pi\right\}
\end{aligned}
$$

The torque is expressed:

$$
\tau=\frac{i_{u} e_{m u}+i_{v} e_{m v}+i_{w} e_{m v}}{\omega_{1}}
$$

where

$$
\begin{aligned}
& e_{m u}, e_{m v}, e_{m w}: \text { induced voltage of phase } u, v \\
& \quad \text { and } w \\
& e_{m u}=\sqrt{2} E_{m} \sin \left(\omega_{1} t\right) \\
& e_{m v}=\sqrt{2} E_{m} \sin \left(\omega_{1} t-2 \pi / 3\right) \\
& e_{m w}=\sqrt{2} E_{m} \sin \left(\omega_{1} t-4 \pi / 3\right)
\end{aligned}
$$

From Eqs ( 6 ) and ( 7$)$, the torque when the subharmonic method is used, $\tau_{s}$, is derived as :

$$
\tau_{s}=\tau_{0}+\sum_{m} \sum_{k} \tau_{h(m, k)}
$$

where

$$
\begin{aligned}
& \tau_{0}= \frac{3 E_{o 1} E_{m}}{\omega_{1} Z_{1}} \\
& \tau_{h}= \frac{3 \sqrt{2} w N E_{d} E_{m}}{2 \omega_{1}} \\
& \times\left\{\frac{A_{(m, k+1)}}{Z_{(m, k+1)}} \cos \left(\omega_{(m, k)} t+\theta_{(m, k+1)}-\phi_{(m, k+1)}\right)\right. \\
&-\frac{A_{(m, k-1)}}{Z_{(m, k-1)}} \cos \left(\omega_{(m, k)} t+\theta_{(m, k-1)}-\phi_{(m, k-1)}\right\} \\
& m= 2 N, 4 N, 6 N, \cdots \quad k=0, \pm 6, \pm 12, \cdots \\
& \tau_{0}: \text { DC component of torque } \\
& \tau_{h(m, k)}: \text { ripple torque component }
\end{aligned}
$$

Eq. ( 8 ) shows that some components of the output current generate no ripple torque components. It is noted that the harmonic components in Fig. 1 (a three-phase three-wire system) are different from those in Fig. 4 (a), and that the difference is negligible because it results in the components canceled in Eq. ( 8 ).

The frequencies of the residual components are expressed as :

$$
\begin{aligned}
& f=g f_{C E}+l^{\prime} f_{1} \cdots \ldots \ldots \ldots \ldots \ldots \ldots \ldots \ldots \ldots(9) \\
& \text { where } g=1,2,3,4,5, \cdots \quad l^{\prime}=\mathrm{t}, \pm 6, \pm 12, \cdots
\end{aligned}
$$

The harmonic components contained in the output voltage, output current and torque in the 


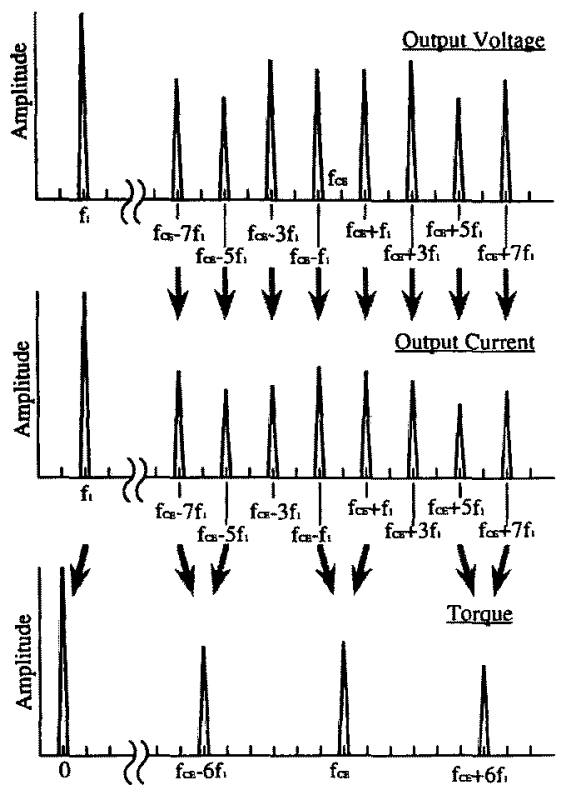

Fig. 5. Relation between harmonic components of output voltage, output cullent and torque.

neighborhood of the equivalent carrier frequency are shown in Fig. 5. The harmonic components of output voltage and output current can be seen at intervals of $2 f_{1}$, and the equivalent carrier frequency is located at the center of them. The ripple torque components are located at the intervals of $6 f_{1}$, because some of the current harmonic components, such as $f_{C E} \pm 3 f_{1}, f_{C E} \pm 9 f_{1}, \cdots$, do not generate ripple torque components. Also, the frequencies are shifted by $f_{1}$ from those of the output voltage.

\section{Ripple torque reduction with harmonic injection}

\subsection{Analysis of harmonic components and the injected component}

When a lower order harmonic is injected into the modulation wave, intersections with the modulation wave and the triangular carrier are shifted forward or backward, resulting in changes of amplitudes and phase angles of the contained harmonic components. Therefore, the injected component can change the harmonic component in an output voltage.
Table 3. Amplitudes of harmonic components in Eq. (10).

\begin{tabular}{|c|c|c|c|}
\hline$m$ & $h$ & $k$ & $A_{(m, k, k)}$ \\
\hline \multirow{2}{*}{$\begin{array}{l}2 N, 4 N \\
6 N, \cdots\end{array}$} & $\begin{array}{l}0, \pm 2 \\
\pm 4, \cdots\end{array}$ & $\begin{array}{l} \pm 1_{i} \pm 3 \\
\pm 5, \cdots\end{array}$ & $\frac{k 4(-1)^{m / 2}}{|k| m \pi} J_{|k|}\left(\frac{m \pi}{2} A_{n}\right) J_{|k|}\left(\frac{m \pi}{2} A_{1}\right)$ \\
\hline & $\begin{array}{l} \pm 1 . \pm 3 \\
\pm 5, \cdots\end{array}$ & $\begin{array}{l}0, \pm 2 \\
\pm 4, \cdots\end{array}$ & $\frac{h 4(-1)^{m / 2}}{|h| m \pi} J_{|h|}\left(\frac{m \pi}{2} A_{n}\right) J_{|k|}\left(\frac{m \pi}{2} A_{1}\right)$ \\
\hline \multicolumn{3}{|c|}{ other combinations } & 0 \\
\hline
\end{tabular}

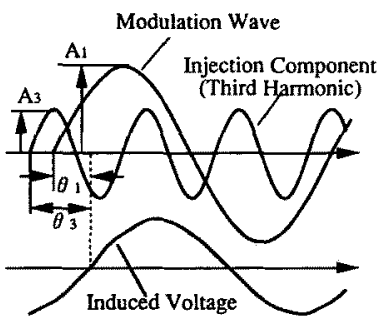

Fig.6. Modulation wave, injection component and induced voltage.

First, the relationship between the harmonic components and the injected component is clarified. Waveforms of the output voltage when the $n$-th harmonic is injected can also be analyzed using the same method as Sec. 3 :

$$
\begin{aligned}
e_{t}= & w N E_{d}\left\{A_{1} \sin \left(\omega_{1} t+\theta_{1}\right)+A_{n} \sin \left(\omega_{n} t+\theta_{n}\right)\right. \\
& \left.+\sum_{m} \sum_{h} \sum_{k} A_{(m, h, k)} \sin \left(\omega_{(m, h, k)} t+\theta_{(m, h, k)}\right)\right\}
\end{aligned}
$$

where

$$
\begin{aligned}
& \omega_{n}=2 \pi f_{n}\left(=2 n \pi f_{1}\right) \\
& \omega_{(m, h, k)}=2 \pi f_{(m, h, k)} \\
& f_{(m, h, k)}=m f_{c}+(n h+k) f_{1} \\
& \theta_{(m, h, k)}=m \theta_{c}+n h \theta_{n}+k \theta_{1} \\
& A_{(m, h, k)}: \text { amplitude index of harmonic compo- } \\
& \quad \text { nent shown in Table } 3 \\
& m, h, k: \text { integers } \\
& A_{n}: \text { modulation index of } n \text {-th harmonic } \\
& f_{n}: \text { frequency of } n \text {-th harmonic } \\
& \theta_{n}: \text { phase angle of } n \text {-th harmonic }
\end{aligned}
$$

Here, the amplitudes of the fundamental and the $n$-th components are decided by modulation indices $A_{1}$ and $A_{n}$ respectively, entirely independent of each other. The frequencies, amplitudes and phase angles of the harmonic components depend on those of both fundamental modulation wave and the injected 
$n$-th harmonic component.

The output current is expressed as follows :

$$
\begin{aligned}
i_{t}= & \frac{1}{Z_{1}}\left\{w N E_{\alpha} A_{1} \sin \left(\omega_{1} t+\theta_{1}-\phi_{1}\right)\right. \\
& \left.-\sqrt{2} E_{m} \sin \left(\omega_{1} t-\phi_{1}\right)\right\} \\
& +\frac{1}{Z_{n}} w N E_{\alpha} A_{n} \sin \left(\omega_{n} t+\theta_{n}-\phi_{n}\right) \\
& +\frac{w N E_{d}}{Z_{(m, h, k)}} \sum_{m} \sum_{h} \sum_{k} A_{(m, h, k)} \\
& \times \sin \left(\omega_{(m, h, k)} t+\theta_{(m, h, k)}-\phi_{(m, h, k)}\right.
\end{aligned}
$$

where

$$
\begin{aligned}
& Z_{n}=\sqrt{R^{2}+\left(\omega_{n} L\right)^{2}} \\
& \phi_{n}=\tan ^{-1}\left(\omega_{n} L / R\right) \\
& Z_{(m, h, k)}=\sqrt{R^{2}+\left(\omega_{(m, h, k)} L\right)^{2}} \\
& \phi_{(m, h, k)}=\tan ^{-1}\left(\omega_{(m, h, k)} L / R\right)
\end{aligned}
$$

The torque with harmonic injection, $\tau_{t}$, is expressed :

$$
\tau_{t}=\tau_{0}+\tau_{n}+\sum_{m} \sum_{h} \sum_{k} \tau_{h(m, h, k)}
$$

where

$$
\begin{aligned}
& \tau_{0}=\frac{3 E_{o 1} E_{m}}{\omega_{1} Z_{1}} \\
& \tau_{n}=\left\{\begin{array}{cc}
0 & (n=3,6,9,12, \cdots) \\
\frac{3 \sqrt{2}}{2} & A_{n} w N E_{d} E_{m} \\
Z_{n} & \\
\times \cos \left\{(n-1) \omega_{1} t+\theta_{n}-\phi_{n}\right\} \\
(n=4,7,10,13, \cdots) \\
-\frac{3 \sqrt{2}}{2} \frac{A_{n} w N E_{d} E_{m}}{Z_{n}} \\
\times \cos \left\{(n+1) \omega_{1} t+\theta_{n}-\phi_{n}\right\} \\
(n=2,5,8,11, \cdots)
\end{array}\right. \\
& \tau_{h}=\frac{3 \sqrt{2} w N E_{d} E_{m}}{2 \omega_{1}} \\
& \times\left\{\frac { A _ { ( m , h , k + 1 ) } } { Z _ { ( m , h , k + 1 ) } } \operatorname { c o s } \left(\omega_{(m, h, k)} t\right.\right. \\
& \left.+\theta_{(m, h, k+1)}-\phi_{(m, h, k+1)}\right) \\
& -\frac{A_{(m, h, k-1)}}{Z_{(c, h, k-1)}} \cos \left(\omega_{(m, k, k)} t\right. \\
& \left.\left.+\theta_{(m, k, k-1)}-\phi_{(m, k, k-1)}\right)\right\}
\end{aligned}
$$

$m=2 N, 4 N, 6 N, \cdots$

$k=0, \pm 6, \pm 12, \cdots$ when $h=0, \pm 2, \pm 6, \cdots$

$k= \pm 3, \pm 9, \pm 15, \cdots$ when $h= \pm 1, \pm 3, \pm 5, \cdots$

$\tau_{0}: \mathrm{DC}$ component of torque

$\tau_{n}$ : torque component caused by the injected component $\tau_{h(m, h, k)}:$ ripple torque component

The torque component $\tau_{n}$, which is generated by the injection component, depends upon the integer $n$. When $n$ is a multiple of three, it does not cause the ripple torque component $\tau_{n}$. The lower the frequency of the injection component, the easier it is to control. Therefore, the third harmonic is selected as the injection component.

Also, when the order of the injection component is odd, the output frequencies of voltage, current and torque are the same as those in Eq. (9) when the subharmonic method is used, which makes the anal. ysis easier. Eq. (12) shows that the amplitudes and the phase angles of the harmonic components can be changed by controlling those of the injected third harmonic $A_{3}$ and $\theta_{3}$.

\subsection{Reduction of dominant ripple torque com-} ponents

From the analysis above, it is clarified that the harmonic components of current and torque can be controlled by regulating the amplitude and the phase angle of the injection component. It is, however, difficult to find the conditions at which all the harmonic components of the current and the torque are reduced. Therefore, it is supposed that the dominant components of the ripple torque are to be minimized, because they directly result in noise and vibration of the load.

From simulation analysis ${ }^{(2)}$, it can be said that the dominant ripple torque components are located at the frequencies $\left(f_{C E}-6 f_{1}\right), f_{C E}$ and $\left(f_{C E}+6 f_{1}\right)$. Let $\tau_{\{f \mathrm{sB}}$ be the ripple torque component of frequency $f_{C E}$. Then, it can be derived using Eq. (12) :

$$
\begin{aligned}
\tau\left(f_{C E}\right)= & \frac{3 \sqrt{2} w N E_{d} E_{m}}{2 \omega_{1}} \delta\left(f_{C E}\right) \sum_{m} \sum_{h} \sum_{k} \\
& \times\left\{\frac { A _ { ( m , h , k + 1 ) } } { Z _ { ( m , h , k + 1 ) } } \operatorname { c o s } \left(\omega_{(m, h, k)} t\right.\right. \\
& \left.+\theta_{(m, h, k+1)}-\phi_{(m, h, k+1)}\right) \\
& -\frac{A_{(m, h, k-1)}}{Z_{(m, h, k-1)}} \cos \left(\omega_{(m, h, k)} t\right. \\
& \left.\left.+\theta_{(m, h, k-1)}-\phi_{(m, h, k-1)}\right)\right\} \cdots
\end{aligned}
$$

where

$$
\delta\left(f_{C E}\right)= \begin{cases}1 & \left(m f_{C}+(3 h+k) f_{1}=f_{C E}\right) \\ 0 & \left(m f_{C}+(3 h+k) f_{1} \neq f_{C E}\right)\end{cases}
$$

Also, the other dominant components $\pi_{\left(f c E^{-6} f_{1}\right)}$ and 


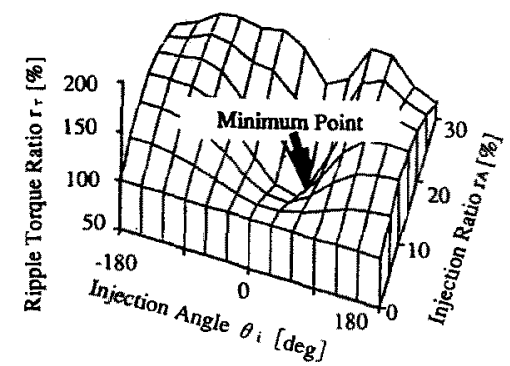

Fig. 7. Characteristics of ripple torque ratio.

$\tau_{\left(U c E+6 f_{1}\right)}$ can be derived in the same way.

Theoretically, there are innumerable combinations which make up the frequency of one of the dominant components. However, because of the characteristics of the Bessel function, the larger the integers $m, h$ and $k$, the smaller the harmonic index $A_{(m, h, k)}$ becomes. Therefore, the amplitudes of dominant components can be given using the integers of the following ranges:

$$
m=2 N,-6 \leqq h \leqq 6,-10 \leqq k \leqq 10
$$

The other amplitude indices are negligible, because they are much smaller.

Here, the injection ratio, injection angle and ripple torque ratio are defined as :

$$
\begin{aligned}
& r_{A}=\left(A_{3} / A_{1}\right) \times 100 \quad(\%) \\
& \theta_{i}=\theta_{3} \quad(\mathrm{deg}) \\
& r_{\tau}=\frac{\Delta \tau_{s}}{\Delta \tau_{t}} \times 100 \quad(\%)
\end{aligned}
$$

where

$$
r_{A}: \text { injection ratio }
$$

$A_{1}:$ modulation index of fundamental component

$A_{3}:$ modulation index of third harmonic

$\theta_{i}$ : injection angle

$r_{\tau}:$ ripple torque ratio

$\Delta \tau_{s}:$ ripple torque with subharmonic method

$\Delta \pi_{t}$ : ripple torque with harmonic injection PWM

Fig. 7 shows an example of the calculated ripple torque when $N=4, A_{1}=0.46$ and $\theta_{1}=41 \mathrm{deg}$, which is the condition to output the rated current in phase with the induced voltage at the output frequency $37.5 \mathrm{~Hz}$. Here, the injection ratio $r_{A}$ ranges from 0 to $30 \%$, so that the amplitude of the modulation

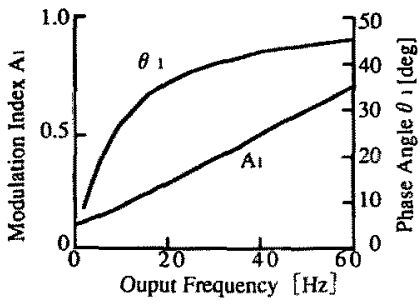

(a) Modulation index and phase angle.

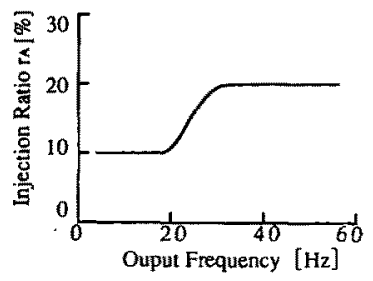

(b) Sutable injection ratio.

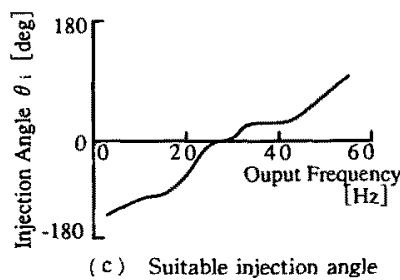

Fig. 8. Suitable injection ratio and injection angle.

wave is smaller than 1 when the modulation index $A_{1}$ is less than about 0.7 . The injection ratio of $20 \%$ and angle of 30 degrees, where the minimum point of ripple torque ratio is located, correspond to the suitable third harmonic to minimize the ripple.

Fig. 8 (a) shows the modulation index and the phase angle when the current is kept at the rated one and in phase with the induced voltage. Figs 8 (b) and (c) show the characteristics of a suitable injection ratio and angle under the conditions of Fig. 8 (a). In a practical use, a table of the suitable injection ratio and angle to the modulation index and the phase angle is calculated in advance.

\section{Simulation results}

Fig. 9 shows the simulation results. Figs 9 (a) and $(c)$ compare the simulation waveforms (the output voltage and current in phase $u$ and the torque), and Figs 9 (b) and (d) show the frequency spectra of the conventional subharmonic method 


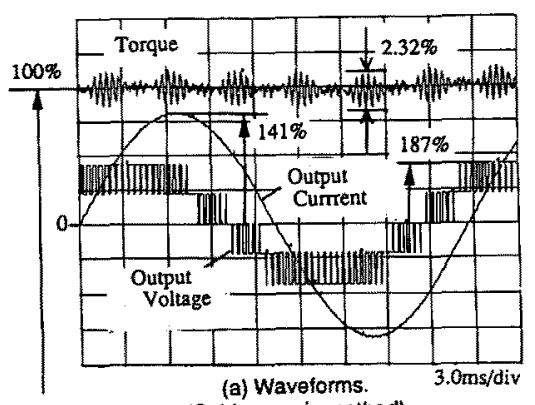

(Subharmonic mothod)
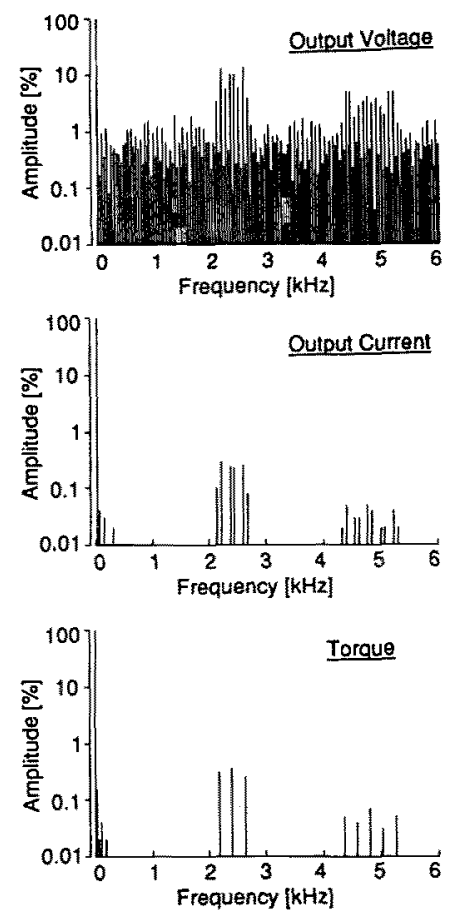

(b) Frequency Spectra (Subharmonic method)

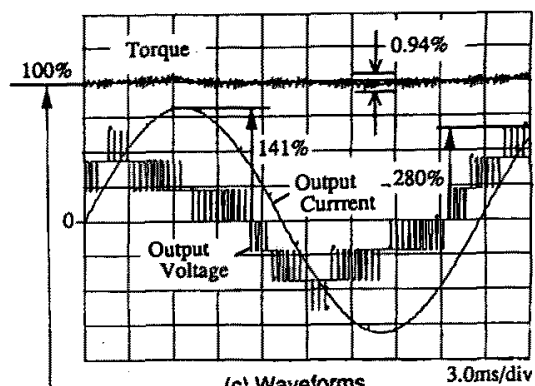

(c) Wavelorms

(Hamonic Injection PWM)
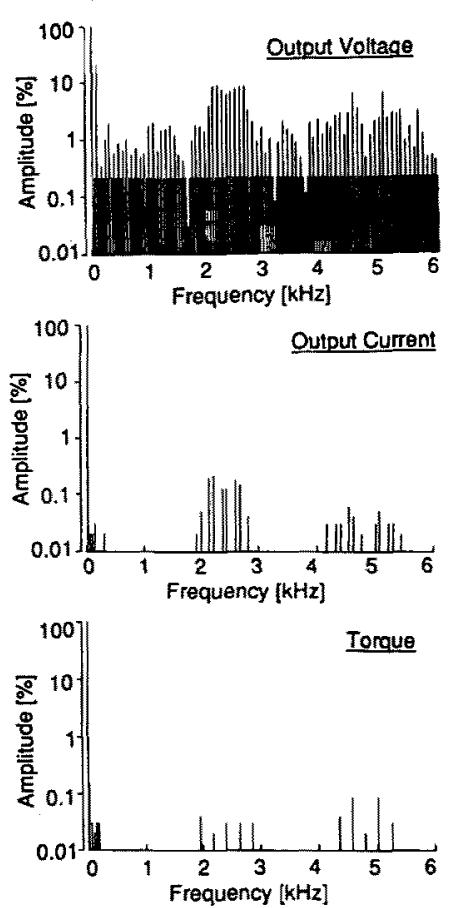

(d) Frequency Spectra. (Harmonic Injection PWM)

Fig. 9. Comparison of simulation results.

Table 4. Simulation constants.

\begin{tabular}{|l|r|}
\hline Output frequency & $37.5 \mathrm{~Hz}$ \\
\hline switching frequency & $300 \mathrm{~Hz}$ \\
\hline N (multiple index) & 4 \\
\hline dc voltage & $93 \%$ \\
\hline resistance & $25 \%$ \\
\hline inductive reactance & $80 \%$ \\
\hline SM induced voltage & $75 \%$ \\
\hline
\end{tabular}

and the harmonic injection PWM, respectively. The simulation constants, shown in Table 4, are normalized considering the induced voltage at the frequency $50 \mathrm{~Hz}$ and the rated current as $100 \%$. The injection ratio and angle in Figs 9 (c) and (d) are suitable (20\%, 30 degrees), as was given in Fig. 7. The ripple torque is expressed as a percentage of the $\mathrm{DC}$ component.

In Figs 9 (a) and (c), both output voltages are multilevel pulse trains, where the harmonics of each unit inverter cancel out. The ripple torque of the subharmonic method is $2.32 \%$, while the harmonic injection PWM successfully reduces the ripple torque to $0.94 \%$. Here, the third harmonic component is not contained in the output current of the 


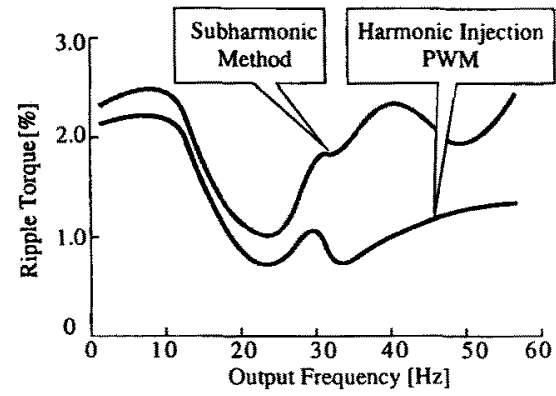

Fig. 10. Comparison of ripple torque.

harmonic injection PWM, as this is a three-phase three-wire system. It is noted that the third harmonic never affects ripple torque reduction with the harmonic injection PWM even when it is contained in the output current.

In the frequency spectra in Figs 9 (b) and (d), harmonic components can be seen near the frequencies $2.4 \mathrm{kHz}$ (the equivalent carrier frequency) and $4.8 \mathrm{kHz}$, which accords with the theoretical analysis. Harmonic current components near the equivalent carrier frequency of the injection PWM are slightly reduced compared with those of the subharmonic method. The dominant ripple torque components of injection PWM are less than $0.1 \%$, while those of subharmonic are about $0.5 \%$.

Fig. 10 shows the effect of ripple torque reduction of the harmonic injection PWM with the condition shown in Fig. 8. At output frequencies under $20 \mathrm{~Hz}$, where the modulation index $A_{1}$ is relatively low, the ripple torque is as large as the conventional subharmonic method. However, at output frequencies higher than $20 \mathrm{~Hz}$, the ripple torque is successfully reduced to less than $1.2 \%$.

\section{Conclusion}

In this paper, a harmonic injection PWM was proposed, which aimed at minimization of the harmonics of the multiple power converter.

First, the Fourier series of PWM voltage when a harmonic component is injected into the reference sinusoid was derived and the relation between the components of voltage, current and torque were clarified. Next, the suitable amplitude and phase angle of the injected third harmonics to minimize the ripple torque were derived. The simulation results showed the effectiveness of the ripple torque reduction.

It is further noted this PWM method is suitable for multiple inverter PWM control, where the switching frequency is relatively low because high power GTOs are used as switching devices.

(Manuscript received Aug. 21, '92,

revised Dec. 24, '92)

\section{References}

(1) M. Hombu: "State-of-the-art of Large Capacity Inverter" (in Japanese), 1992 National Convention Record I. E. E. Japan, S. 8

(2) T. Sukegawa, K Kamiyama, J. Takahashi, T. Ikini \& M. Matsutake: "A Muitiple PWM GTO Line-side Converter for Unity Power Factor and Reduced Harmonic", Conf. Rec. 1991 IEEE-IAS Annual Meetig

(3) N. Kawakami, Y. Jifuku, S. Ueda, M. Hombu \& A. Ueda: "A Large Capacity GTO Inverter with Low-Loss Snubber Circuits", Conf. Rec. 1991 IEEE-IAS Annual Meeting

(4) K. Suzuki, T. Nakajima, H. Konishi \& T. Nakamura: "A Study of Control System for Self-Commutated Converter Compensator" (in Japanese) Tran IEE Japan 112-B, No. 1 (1992)

(5) K. Nakata, S. Ito \& K Nakamura: "Analysis of Output Harmonics of Multiple Inverter for LSM Drive", (in Japanese) 1990 Convention Record I.E. E. Japan S. P.C., SPC- $90 \div 40$

(6) S. Ito, K. Nakata \& K. Nakamura: "Harmonic Characteristics of Multiple Inverter for LSM Drive when Third Harmonic Component of Induced Voltage is Considered", (in Japanese) 1990 Convention Record I. E. E. Japan S. P. C. $\mathrm{SPC}-90-41$

(7) S. Tadakuma, S. Tanaka, H. Inoguchi, Y. Tanoue, H. Ikeda \& S. Kaga: "Consideration on Large Capacity PWM Inverter for LSM Drive". Int. Power Electron. Conf. Rec., Vol. 1, 1990

(8) Y. Hosokawa, T. Okajima, J. Kitano, N. Morishima, M. Tanaka \& Y. Yonehata: "The Control Scheme of the PWM Multiple Inverter for LSM Drive", Conf. Rec. 1990 IEE Japan IAS

(9) H. Le-Huy \& L. A. Dessaint: "An Adaptive Current Control Scheme for PWM Synchronous Motor Drives: Analy. sis and Simulation", IEEE Trans. Power Electron. 14, No. 4 (1989)

(10) Y. Iwaji \& S. Fukuda : "PWM pulse pattern optimization for sinusoidal inverters", Int. Power Electron. Conf. Rec., 1990

(11) M.A. Boost \& P.D. Ziogas: "State-of-the-Art Carrier PWM Techniques: A Critical Evaluation", IEEE Trans. Industn. Applic., 24, No. 2 (1988)

(12) R. Bonert \& R.S. Wu: "Improved Three Phase Pulsewidth Modulation for Overmodulation". Conf. Rec. 1984 IEEE-IAS Annual Meeting

(13) T. Kume, S. Sonoda, T. Kai, H. Ryu \& E. Watanabe: "Supression of Lower Harmonics in PWM Inverter", (in Japanese) 1991 National Convention I. E. E. Japan-Industry Applications Society"., No. 150

(14) "Semiconductor Power Conversion", (in Japanese) IEE Japan, Tokyo, 1987, Chap. 6 


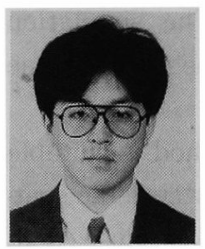

Satoru Ito (Member)

He was born in Hokkaido on November 15, 1964. He received the B.S. and M.S. degrees in electrical engineering from Hokkaido University in 1987 and 1989, respectively. He joined in 1989. Since 1989, he worked at Hitachi Research Laboratory, Hitachi, Ltd. in 1989 and he has been engaged in the research and development of traction systems and multiple inverters for LSM drive.

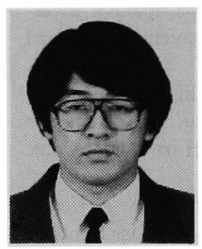

\section{Kiyoshi Nakata (Member)}

He was born on August 28, 1960. He received the B.S. and $M$.S. degrees in elecrtical engineering from Tokyo Denki University in 1983 and 1985, respectively. He joined Hitachi Research Laboratory, Hitachi, Ltd. in 1985, where he has been engaged in the research and development of traction systems.

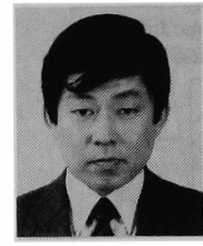

Shigeta Ueda (Member)

He was born in Hokkaido on April 25 , 1956. He received the B.S. and M. S. degrees in electrical engineering from Hokkaido University in 1979 and 1981, respectively. He joined Hitachi Research Labora. tory, Hitachi, Ltd. in 1981. Since 1981, he has been engaged in research of power converters using GTO's for industrial drives and electric power systems. $\mathrm{He}$ is currently a Senior Researcher.

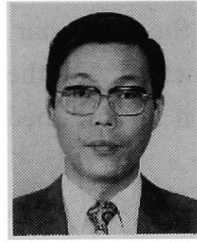

\section{Kiyoshi Nakamura (Member)}

He was born on July 24, 1944. He received the $B$.S. degree in electrical engineering from Waseda University in 1967. He joined Hitachi Research Laboratory, Hitachi, Ltd. in 1967, and he has been engaged in the research and development of traction systems for rolling stock, drive systems of elevators, and power electronics. He is currently a Chief Researcher. 